\title{
$\underline{\mathbf{P}-123}$
}

\section{Evaluation of the Acute, Sub-Chronic Toxicity and the Antioxidant Effect of Ellagic Acid in Smoker and Non Smoker an Experimental Study in Rats}

\author{
Mazen.M.Jamil, Fouad Hussain Al-Bayaty ${ }^{*}$, Farha Ariffin Rami Al-Batran
}

Faculty of Dentistry, Universiti Teknologi MARA, 40450 Shah Alam, Malaysia; E-mail: fouad@salam.uitm.edu.my

Objective: The aim of this study is to investigate the possible protective antioxidant role of the Ellagic acid (EA) on smoker and non Smoker rats using biochemical and antioxidant approaches and to evaluate the acute and sub chronic toxicity test of Ellagic Acid.

Materials and Methods: Twenty four Adult male Sprague-dawley (SD) rats were randomly divided in four groups (6 rats each group). Group 1 control, Group 2 received Ellagic Acid, groups 3 and 4 were injected subcutaneously with (0.5 mg/kg) Nicotine (Ni) for one month to convert the animals into smoker. Then, group 4 was gavaged with EA (50 mg/kg) for 30 days using gastric tube. Animal sacrificed after 30 days. Antioxidants enzyme parameters, Superoxide Dismutase (SOD), Malondialdehyde (MDA), Catalze (CTA), Nitric oxide (NO) levels were evaluated in serum and liver, kidney, heart homogenate. Cotinine level was measured in serum.

Results: Ellagic Acid induced an increase in the free radical-scavenging enzyme activity (SOD), and Catalze (CTA) levels in non nicotinic rats. While in nicotinic rats the results demonstrated significant decrease in (SOD), and Catalze (CTA) levels. NO level was significantly higher in Ni group compare to other groups. MDA level was higher in nicotinic group than other groups. There was a significant increase in the cotinine levels in nicotinic group. While it is significantly decrease in Ellagic acid treated group.

Conclusions: This study showed that Ellagic Acid is able to significantly accelerate the activities of the antioxidant. No toxicity of the Ellagic acid was demonstrated to a dose of $1000 \mathrm{mg} / \mathrm{kg}$ body weight.

Keywords: Ellagic acid, Antioxidant, nicotine, Rat. 